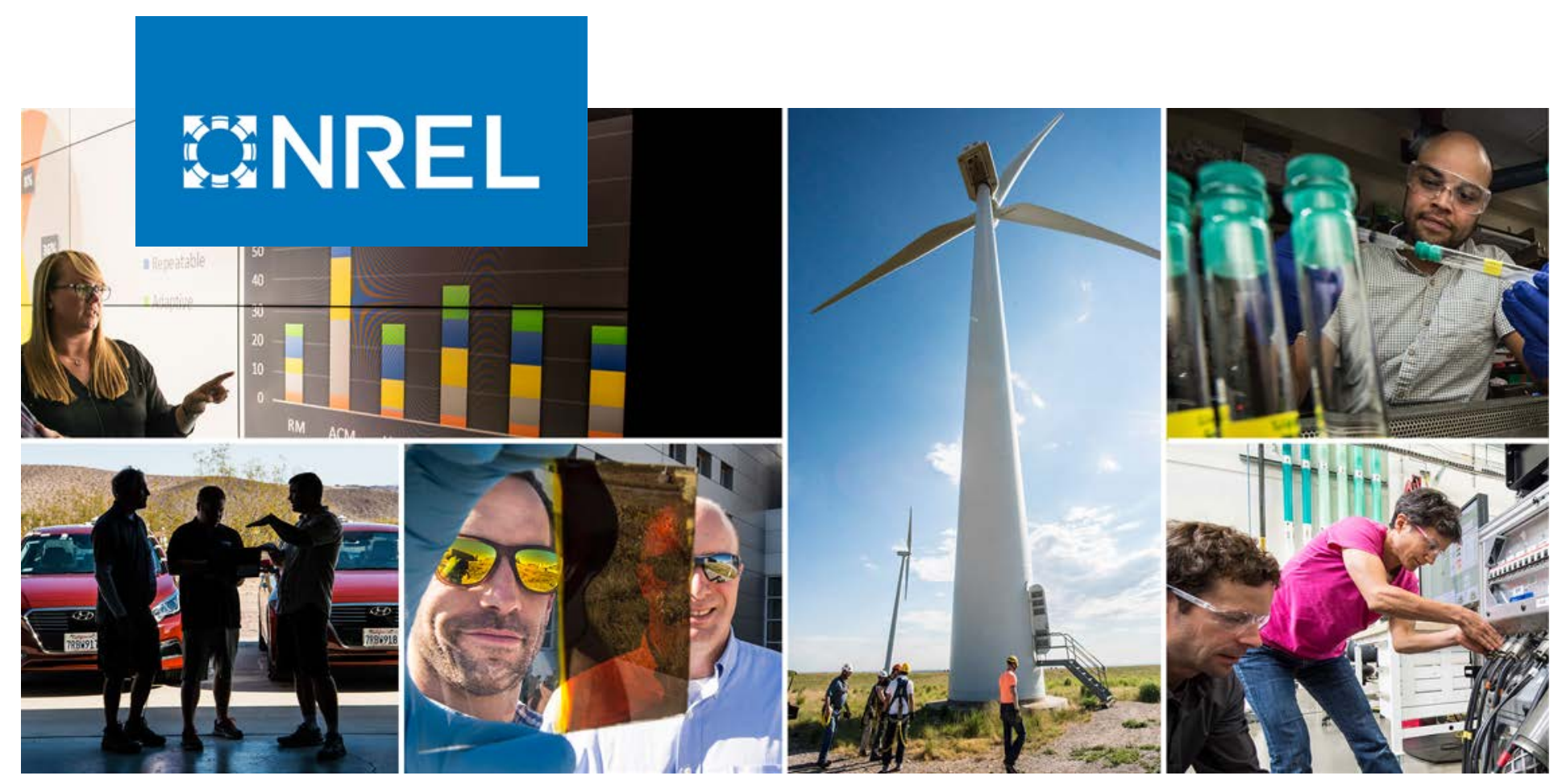

\title{
Informative Background on the Interoperability Requirements in IEEE Std 1547-2018
}

Michael Ingram, Rasel Mahmud, and David Narang

National Renewable Energy Laboratory

NREL is a national laboratory of the U.S. Department of Energy Office of Energy Efficiency \& Renewable Energy

Operated by the Alliance for Sustainable Energy, LLC

This report is available at no cost from the National Renewable Energy Laboratory (NREL) at www.nrel.gov/publications.
Technical Report

NREL/TP-5D00-77959

July 2021 


\section{FANEL}

\section{Informative Background on the Interoperability Requirements in IEEE Std 1547-2018}

Michael Ingram, Rasel Mahmud, and David Narang

National Renewable Energy Laboratory

\section{Suggested Citation}

Ingram, Michael, Rasel Mahmud, and David Narang. 2021. Informative Background on the Interoperability Requirements in IEEE Std 1547-2018. Golden, CO: National Renewable Energy Laboratory. NREL/TP-5D00-77959.

https://www.nrel.gov/docs/fy21osti/77959.pdf.

NREL is a national laboratory of the U.S. Department of Energy Office of Energy Efficiency \& Renewable Energy Operated by the Alliance for Sustainable Energy, LLC

This report is available at no cost from the National Renewable Energy Laboratory (NREL) at www.nrel.gov/publications.

Contract No. DE-AC36-08GO28308
Technical Report NREL/TP-5D00-77959 July 2021

National Renewable Energy Laboratory 15013 Denver West Parkway Golden, CO 80401 303-275-3000 • www.nrel.gov 


\section{NOTICE}

This work was authored by the National Renewable Energy Laboratory, operated by Alliance for Sustainable Energy, LLC, for the U.S. Department of Energy (DOE) under Contract No. DE-AC36-08GO28308. Funding provided by U.S. Department of Energy Office of Energy Efficiency and Renewable Energy Solar Energy Technologies Office Award Number 34808. The views expressed herein do not necessarily represent the views of the DOE or the U.S. Government.

This report is available at no cost from the National Renewable Energy Laboratory (NREL) at www.nrel.gov/publications.

U.S. Department of Energy (DOE) reports produced after 1991 and a growing number of pre-1991 documents are available free via www.OSTI.gov.

Cover Photos by Dennis Schroeder: (clockwise, left to right) NREL 51934, NREL 45897, NREL 42160, NREL 45891, NREL 48097, NREL 46526.

NREL prints on paper that contains recycled content. 


\section{Preface}

The revised Institute of Electrical and Electronics Engineers (IEEE) 1547-2018, Standard for Interconnection and Interoperability of Distributed Energy Resources with Associated Electric Power Systems Interfaces, was published in April 2018. This standard is one of the foundational documents in the United States needed for integrating distributed energy resources (DERs), including solar energy systems, with the electric distribution grid.

The revised standard contains 11 chapters (clauses) and 8 annexes that comprise 136 pages. The revision is significantly different from the 2003 version, and it contains new concepts and new technical requirements. Each clause specifies information or requirements that apply to certain aspects that are important to the interconnection of DERs to the electric power system. Implementing the requirements necessitates a careful study of the underlying technical concept and requires appropriate information to calculate relevant settings and configurations.

Various stakeholders have different roles in implementing the standard, and portions of the standard are directed toward a specific audience who must possess specialized information and technical training to use and apply the requirements.

This document aims to (1) summarize a specific portion of the standard as concisely as possible and (2) provide the reader with introductory knowledge and information to support the utilization of the requirements.

Note that the narrative on the implementation and configurability of the requirements reflects the authors' interpretations, which in some instances might differ from one person to another, especially during this early stage of implementation; therefore, this work is intended to supplement the existing ${ }^{1}$ and growing body of knowledge ${ }^{2}$ across the U.S. electric sector on the use and application of this important standard.

${ }^{1}$ Additional educational material can be found at https:/www.nrel.gov/grid/ieee-standard-1547/.

${ }^{2}$ See the IEEE Standards Coordinating Committee 21 for more resources:

https://sagroups.ieee.org/scc21/standards/1547rev/. 


\section{Acknowledgments}

This material is based on work supported by the U.S. Department of Energy Office of Energy Efficiency and Renewable Energy under the Solar Energy Technologies Office Award Number 34808.

The authors thank Jason Allnutt, Jens C. Boemer, Michele Boyd, Robert Harris, Bob Fox, Brian Lydic, Joe Palladino, Emma Raszmann, John Shenot, Akanksha Singh, Ravi Subramaniam, and Kiera Zitelman for their review and valuable comments.

The authors are also grateful to the U.S. Department of Energy Office of Energy Efficiency and Renewable Energy Solar Energy Technologies Office Strategic Analysis and Institutional Support Program Manager Michele Boyd, Robert Reedy and Jeremiah Miller, as well as Systems Integration Program Manager Guohui Yuan for their guidance and support. 


\section{List of Acronyms}

AGIR

CSIP

DER

DNP3

EPS

FERC

IEC

IEEE

IP

NERC

PCC

PV

SEP 2.0

TCP

TLS

UDP

$\mathrm{VAR}^{3}$

WECC
Authority Governing Interconnection Requirements

Common Smart Inverter Profile

distributed energy resource

Distributed Network Protocol 3

electric power system

Federal Energy Regulatory Commission

International Electrotechnical Commission

Institute of Electrical and Electronics Engineers

Internet Protocol

North American Electric Reliability Corporation

point of common coupling

photovoltaic

Smart Energy Profile 2.0

Transmission Control Protocol

Transport Layer Security

User Datagram Protocol

volt-ampere reactive

Western Electricity Coordinating Council

${ }^{3}$ IEEE Std 270-2006 uses the name and symbol var for reactive power, not VAR, which is used in this document. (See IEEE Std 270-2006 definitions 3.251 for reactive power and 3.327 for var.) 


\section{Table of Contents}

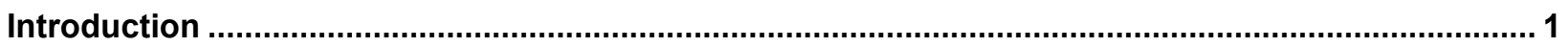

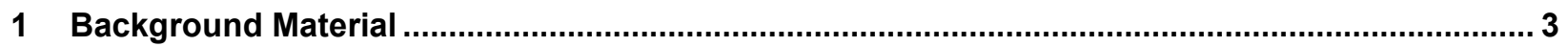

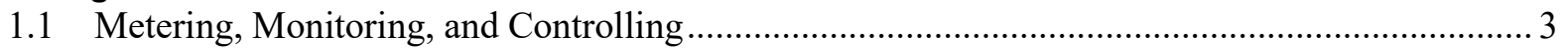

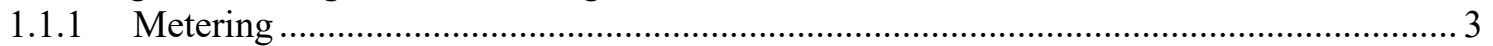

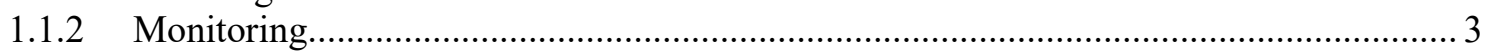

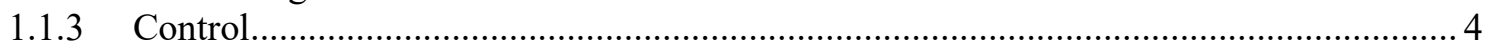

1.1.4 Applications of Monitoring, Information Exchange, and Control ..............................5

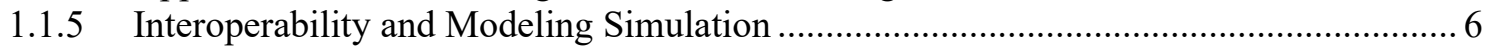

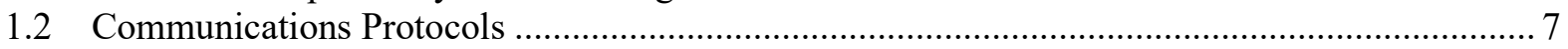

1.2.1 SunSpec Modbus ........................................................................................... 7

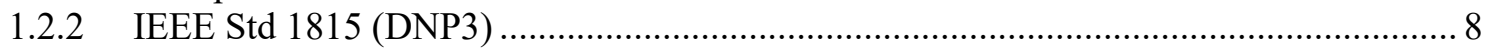

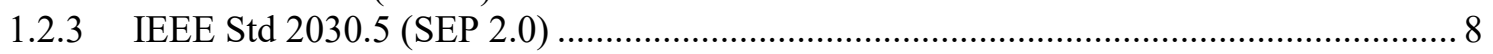

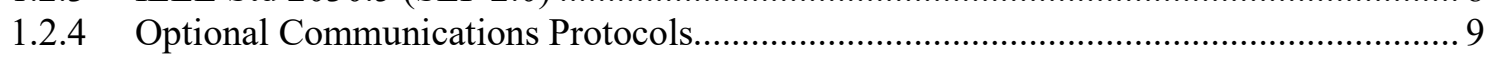

1.3 Communications Architecture......................................................................... 9

2 Summary of Interoperability Requirements in IEEE Std 1547-2018 ....................................

2.1 Information Exchange (Clause 10)..................................................................... 10

2.2 Management Control Capability Requirements .............................................................. 10

2.3 Security Considerations........................................................................................ 11

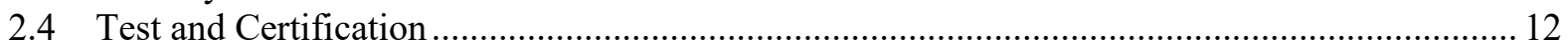

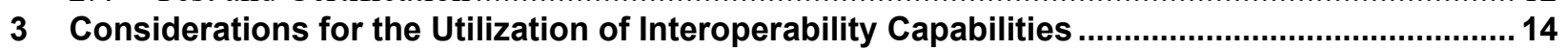

3.1 Interoperability of Distributed Energy Resources and What?........................................... 14

3.2 Utility Requirements for Monitoring and Control.......................................................... 14

3.3 Aggregators/Distributed Energy Resource Grid Services Providers.................................... 15

3.4 Bulk Power System Data Needs............................................................................... 15

3.4.1 Balancing Authorities.................................................................................... 16

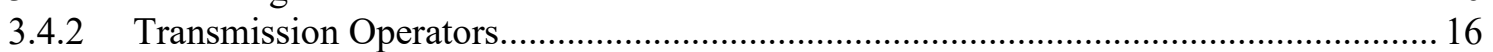

3.4.3 Transmission Planners and Planning Coordinators ................................................ 16

3.5 Screening Criteria for Monitoring and Control .............................................................. 17

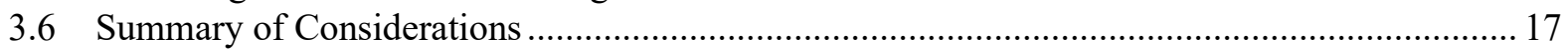

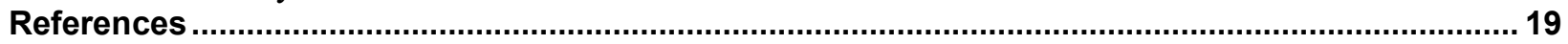




\section{List of Figures}

Figure 1. Hierarchy of communications and interoperability of DERs ........................................... 7

Figure 2. Hierarchical architecture for DER communications..................................................... 9

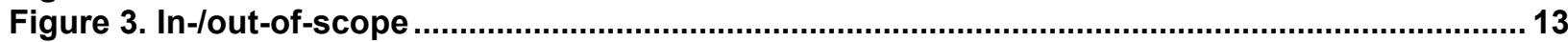




\section{Introduction}

This document provides supplementary information to help stakeholders ${ }^{4}$ apply the interoperability requirements specified in Clause 10 of the revised Institute of Electrical and Electronics Engineers (IEEE) 1547-2018, Standard for Interconnection and Interoperability of Distributed Energy Resources with Associated Electric Power Systems Interfaces (IEEE Std 1547-2018).

The term interoperability as used in IEEE Std 1547-2018 follows the IEEE Std 2030 definition: "The capability of two or more networks, systems, devices, applications, or components to externally exchange and readily use information securely and effectively" (IEEE 2018). This clause did not exist in the previous (2013) version of the standard, and the interpretation of the term was left for utilities and DER developers to negotiate based on system needs.

The full title of Clause 10 is "Interoperability, information exchange, information models, and protocols." Note that although the clause contains requirements for mandatory capabilities of distributed energy resources (DERs) that the manufacturer must ensure, the utilization of interoperability capabilities and methods for implementation are decisions for local jurisdictions. $^{5}$

Emergency and standby DERs are exempt from the interoperability requirements specified in Clause 10. Requirements for communications network specifics as well as for communications inside a DER facility are outside the scope of IEEE Std 1547-2018. In addition, the standard does not address other important considerations, such as the cybersecurity of communications protocols and infrastructure.

Clause 10 is directed primarily toward DER manufacturers; however, if interoperability capabilities are used, additional stakeholders include the area electric power system (EPS) operator, the DER operator, the testing agency, and the commissioning agency. In some jurisdictions, guidance and requirements for the utilization of interoperability capabilities and methods for implementation are also given by the Authority Governing Interconnection Requirements (AGIR). ${ }^{6}$ Depending on the implementation, additional stakeholders might include

\footnotetext{
${ }^{4}$ Although DER manufacturers are a primary stakeholder, their concerns are with equipment design and manufacturing. This document is intended to provide an overall summary for stakeholders involved in the application of IEEE Std 1547-2018.

${ }^{5}$ IEEE Std 1547-2018 Footnote 117 states: "This standard mandates these interoperability capabilities; however, how they are implemented, recorded, and reported is up to local jurisdictions and should be addressed in those processes and procedures (such as interconnection agreements)" (IEEE 2018).

${ }^{6}$ The term Authority Governing Interconnection Requirements (AGIR) is defined in IEEE Std 1547-2018 as a "cognizant and responsible entity that defines, codifies, communicates, administers, and enforces the policies and procedures for allowing electrical interconnection of DER to the Area EPS. This may be a regulatory agency, public utility commission, municipality, cooperative board of directors, etc. The degree of AGIR involvement will vary in scope of application and level of enforcement across jurisdictional boundaries. This authority may be delegated by the cognizant and responsible entity to the Area EPS operator or bulk power system operator. NOTE-Decisions made by an authority governing interconnection requirements should consider various stakeholder interests,
} 
DER aggregators, ${ }^{7}$ bulk power system operators, the regional reliability coordinator, and the DER operator.

No specialized fluency and training are required to understand the material in the subclause; however, the fluency level needed to implement ${ }^{8}$ these interoperability capabilities might vary significantly ${ }^{9}$ depending on whether to use the capabilities as specified or if additional requirements are added.

This document aims to provide a high-level summary of the context and background concepts for requirements related to interoperability. This document is intended as a supplement to material already published or in development. ${ }^{10}$ This document is not intended as an exhaustive resource on technical implementation; rather, topics are presented at a level that is appropriate to serve individuals who require an introduction or technical refresher to the material.

including but not limited to Load Customers, Area EPS operators, DER operators, and bulk power system operator" (IEEE 2018).

7 In IEEE Std 1547-2018, “DER aggregator" falls under the term DER managing entity.

${ }^{8}$ Guidance on the implementation of interoperability capabilities might require substantial effort. As an example, consider the process for the California Rule 21 Smart Inverter Working Group on the implementation of communications (Phase II): https://www.cpuc.ca.gov/general.aspx?id=4154.

${ }^{9}$ For a summary of requirements - including defaults, options, and key decisions - see the Clause-by-Clause Summary of Requirements in IEEE Standard 1547-2018, https:/www.nrel.gov/docs/fy20osti/75184.pdf.

${ }^{10}$ For example, the expected revision to IEEE Std 1547.2, Application Guide for IEEE Std 1547. 


\section{Background Material}

\subsection{Metering, Monitoring, and Controlling}

Virtually all energy planning and distribution grid management functions require knowledge of basic electrical quantities, such as active and reactive power, voltage, and frequency. ${ }^{11}$ The increase in intelligent grid-edge devices has also increased the need for interoperability ${ }^{12}$ and information exchanges among devices and systems. Traditional distribution functions - such as planning, operation, and protection — need these types of information, and they are critical for newer functions, such as the provision of ancillary services and forecasting load and distributed generation.

\subsubsection{Metering}

The most common measurement on the distribution network is metering. With respect to interconnected generation, metering is a means of determining the energy production $(\mathrm{kWh})$ of DERs throughout time (e.g., via monthly meter reads or 15-minute data). Generally, these measurements are captured by a billing/production metering system (meter plus backhaul). The metering system is subject to jurisdictional (state, local, utility, etc.) compliance because of the financial implications of the information developed from the data. Even "basic" metering systems are capable of production performance assessments, such as peak generation and interval energy (typically every 15 minutes). Newer metering systems might provide the capability to measure additional parameters, such as voltage and reactive power. Higher resolution metering provides an enhanced level of situational awareness and the ability to plan with increased confidence and accuracy.

\subsubsection{Monitoring}

Monitoring refers to near-real-time methods that communicate system status, output level (kW), etc., to the utility and possibly to other stakeholders. Monitoring aids distribution planning departments in performing a variety of longer-term functions, including asset management, capacity planning, and forecasting (e.g., transformer sizing, phase balancing, load planning, and protection review). By increasing the fidelity of and supporting off-line models, monitoring also improves future interconnection decisions (i.e., hosting capacity determinations). In distribution operation departments, monitoring data are used for load management (e.g., native load determination, reconfiguration planning, fault restoration). As the monitoring system's veracity, velocity, volume, and variety better match the complexity of the physical system, monitoring enables higher order applications. Advanced distribution management system applications can inform the operation of other controllable equipment (e.g., volt/volt ampere reactive [VAR] optimization). High penetration levels of DERs ultimately necessitate data to support resource planning at the level of the balancing authority or independent system operator because better state estimates are needed to forecast reserves, ramping, etc. Sub-transmission planning and

\footnotetext{
${ }^{11}$ Portions of this background material were previously published in Supplemental Information for New York State Standardized Interconnection Requirements, https:/www.nrel.gov/docs/fy180sti/70183.pdf.

12 IEEE Std 1547-2018 defines interoperability as "The capability of two or more networks, systems, devices, applications, or components to externally exchange and readily use information securely and effectively" (IEEE 2018).
} 
operations might require monitoring for certain circuits affected by high penetration levels of DERs.

\subsubsection{Control}

The term control - in general, with respect to distribution systems - could refer to a variety of means of affecting the behavior of distribution equipment, including DERs. This could include, for example, the control of protective devices, switches, and breakers and managing DER power output and other functions.

One type of control is direct utility control of a load-break element in-line with a DER grid connection (which might include utility-owned protection). Similar to monitoring, control serves a host of functions; the most important is safety. Generally, control ensures the safe practice of distribution maintenance functions (e.g., hot line tagging/arc flash mitigation). Functions of distribution operations enabled by control include feeder reconfiguration and restoration. When coupled with possible additional protection applied at the point of common coupling (PCC) control element, reductions in DER downtime are potentially achievable with certain control schemes.

Utilities often perceive control as necessary to dispatch/curtail photovoltaics (PV) to ensure reliability, efficiency, and safety (especially to ensure anti-islanding protection) in normal and alternate circuit configurations. Direct control of a PV system — with the ability to disconnect it - is considered a means to maintain system safety and reliability during abnormal local system conditions. Advanced control capability is a consideration to meet future requirements for DER markets, volt/VAR, and frequency control (Adams 2017). For these distribution operation functions, it is advantageous to add real-time monitoring of additional locations along the feeder, generation, and load centers and of the connection status of key generators and loads.

Control has traditionally been achieved through supervisory control and data acquisition systems that monitor various parameters at key locations, beginning at the source substation and continuing through various locations along the circuit. Control of other assets might also be required, such as part of lockout procedures during maintenance or other grid operations.

Controlling the DER/plant directly by using externally derived set points to meet specific control objectives is considered advanced control. Examples of interoperable advanced control include the capability of DERs to respond to inputs for distribution-level services, such as voltage regulation, VAR support, scheduling, controlled curtailment, and capacity management. Advanced control enables DERs to be actively dispatched or to participate in services at the level of the independent system operator (e.g., providing reserves, following automatic generator control), potentially improving grid operations at high penetration levels. 
Potentially, under a high penetration level of DERs, an independent system operator could use advanced control to mitigate excess generation (curtailment). In the future, grid-supportive inverter technology might provide additional ancillary services that require advanced control. ${ }^{13}$

\subsubsection{Applications of Monitoring, Information Exchange, and Control}

Distribution planning and operations have different needs from distribution protection regarding monitoring, information exchange, and control.

Distribution planning functions - such as upgrades to equipment and infrastructure to meet increasing customer demand-require measurements at key points on the system and under specific conditions, such as during peak load. Distribution operation functions - such as load management, routine maintenance, and response to unplanned outages - require robust, real-time situational awareness to execute successfully. Some distribution system management techniques also require a certain degree of control, which could be manual or automated, depending on the equipment and need. In addition to providing information for distribution system operators, measurements can provide useful inputs for further modeling and simulation, especially related to the planning and the integration of distributed generation.

Distribution planning, which considers a relatively long time frame (months to years), might be adequately addressed by well-placed and well-timed measurements of load and generation. Traditionally, measurements taken by an energy management system at the feeder head (substation) and from other equipment, such as voltage regulators and capacitor banks on the feeder, have provided adequate information for this function. As the mix of distributed generation and controllable loads (current and forecasted) on feeders increases, however, additional measurements (augmented with modeled forecasts) interior to the feeder at key load and generation centers and at key times (such as during peak load and during minimum daytime load) become necessary. In future scenarios, timely information exchange among the distribution service provider and external entities, such as aggregators or regional planners, also becomes more important.

Distribution operation functions require robust, real-time situational awareness to address concerns related to load management, routine maintenance, unplanned outages, fault restoration, and system reconfiguration, or upgrades on the distribution line or at customer premises. For these functions, it is advantageous to add real-time monitoring of additional locations along the feeder, generation, and load centers, and the connection status of key generators and loads. Control of other assets might also be required, such as lockout during maintenance or for other grid operations. A special case might be communications requirements to update firmware for key assets. To avoid unnecessary expenses for equipment, infrastructure, and staff, some consideration should be given to optimizing the monitoring strategy. Some information exchange

13 IEEE Std 1547-2018 defines "open-loop response time" and specifies requirements for performance (i.e., Tables $8,10,24,31,34,38)$ in response to a local EPS quantity (e.g., voltage or frequency), but these requirements for open-loop response time should not be confused with DER response to a communications signal. Subsection 4.6 .3 allows up to $30 \mathrm{~s}$ for an "invoked action to begin" following an input to the local DER communications interface (IEEE 2018). 
might be necessary between the distribution service provider and external entities - for example, in cases of feeder boundary reconfiguration or notification of outages.

Distribution protection requirements for each protection element are planned to provide adequate coordination under various fault conditions and are then programmed into each protection element to provide a coordinated response. The protection devices respond automatically based on grid conditions at the point of connection. Note that inverter-based systems also have an automatic response to fault conditions. Under fault conditions, protective functions generally operate too quickly for any effective human intervention based on monitoring or control.

Other considerations, such as power quality, might require measurements and/or monitoring at key locations and facilities. Power quality is a complex interaction of load, line, and, today, distributed generation. The seasonal nature of the solar resource, for example, coupled with short-term variability in load and generation contribute to the difficulty in pinpointing the root cause of power quality anomalies. It is advisable to augment field data with software modeling and simulation of these situations to determine the location and size threshold for required monitoring.

\subsubsection{Interoperability and Modeling Simulation}

To effectively dispatch ${ }^{14}$ DERs (market operation), monitoring and control strategies must include balanced investment as well as modeling and simulation of load and generation. Adequate and timely information exchange among energy service providers and aggregators, adequate control of dispatchable assets, and the degree to which the modeling and simulation of load and generation can support the monitoring framework all must also be considered.

Integrating the monitoring and modeling platforms could help optimize the investment in equipment and resources needed for reliable, safe, resilient, and affordable electric service to meet the significant changes in generation, distribution, and management of energy. This integration will depend on the extent to which diverse equipment and systems are interoperable.

Generally, the requirements for DER monitoring and control are a function of the following:

- DER project size: Monitoring is applied first, and monitoring and control are applied at higher project levels.

- Voltage level and overall aggregate DER penetration of a circuit

- Market requirements: These often supersede interconnection monitoring and control requirements (i.e., participating in a wholesale market often requires visibility from the level of the independent system operator).

- DER asset ownership: This could influence required monitoring and control (particularly control) because of safety concerns.

${ }^{14}$ Dispatch is the inclusion of a generator's output onto the electric grid by an authorized scheduling entity to produce energy at the lowest cost to reliably serve consumers, recognizing any operational limits of generation and delivery (U.S. Congress 2005; DOE 2015). 


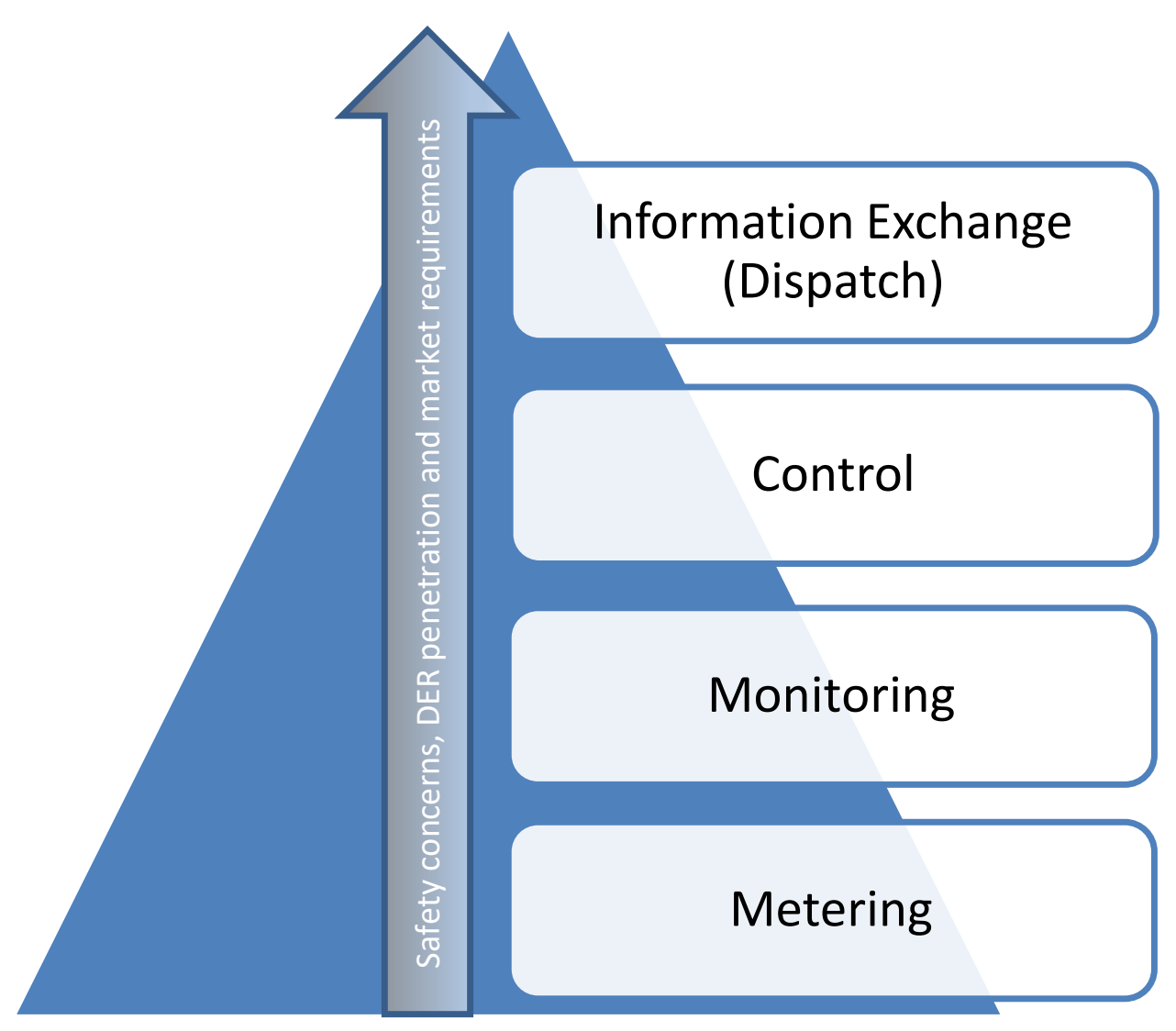

Figure 1. Hierarchy of communications and interoperability of DERs

\subsection{Communications Protocols}

Communications and information protocols were not addressed in the prior (2003) version of IEEE Std 1547 and were left for utilities and DER developers to negotiate based on system needs. This created challenges for the equipment suppliers, which traditionally used proprietary protocols in their equipment and had little guidance on required protocols, as well as for utilities, which frequently use a combination of communications and information protocols. IEEE Std 1547-2018 brings new requirements for communications protocols that must be available at the local DER communication interface, ${ }^{15}$ and the DER must support one of the following protocols:

- SunSpec Modbus

- IEEE Std 1815 (Distributed Network Protocol 3 [DNP3])

- IEEE Std 2030.5 (Smart Energy Profile 2.0 [SEP 2.0]).

\subsubsection{SunSpec Modbus}

Modbus is a control protocol that was originally developed by Modicon (now part of Schneider Electric) in 1979. Because of its long life and simple construction, it is widely employed across a broad range of industrial control systems, including the digital infrastructure for controlling

\footnotetext{
15 The phrase "communication interface" refers to the local DER communication interface specified in IEEE Std 1547-2018, which is defined as "A local interface capable of communicating to support the information exchange requirements specified in this standard for all applicable functions that are supported in the DER (IEEE 2018).
} 
power systems and DERs. Originally a serial protocol, Modbus has been extended to work over Transmission Control Protocol/Internet Protocol (TCP/IP).

Because Modbus was developed before modern networking protocols and equipment, it was designed to be simple and fast, but it lacked native security measures (trade-off). The Modbus protocol, which is employed by SunSpec DER devices, is sometimes cited as a "weak link" in the security chain because of its lack of security (Lai et al. 2017). Yet there is now a standard for Modbus Transport Layer Security (TLS) over TCP/IP for systems that require security (Modbus 2018).

SunSpec describes the information models and data exchange formats used with DER systems and includes nameplate information, monitoring data structure, and advanced grid support functions (IEC 2012) required by IEEE Std 1547-2018 (Clause 10).

\subsubsection{IEEE Std 1815 (DNP3)}

DNP3 represents a more complicated data structure than Modbus, and it allows the capability to define different priority levels for different variables so that high priority messages are given precedence over lower priority messages. This approach aids network communications traffic scheduling. Additionally, DNP3 messages are time-stamped, which is an important feature for analyzing problems or events. For these reasons, DNP3 is predominant in many utility power systems communications (e.g., supervisory control and data acquisition).

Like Modbus, DNP3 was not originally designed with any native security features; however, an updated version, released in 2007 , added secure authentication mechanisms using a challengeresponse exchange between devices (actors) - that is, if Device A makes an access or data request to Device B, Device B will issue a challenge. It is then the responsibility of Device A to prove its identity (authentication) and sufficient privilege level (access control). IEEE Std 1815 recommends the application of International Electrotechnical Commission (IEC) 62531 (including TLS) for a secure implementation of DNP3.

\subsubsection{IEEE Std 2030.5 (SEP 2.0)}

SEP 2.0, based on ZigBee Smart Energy 1.X, outlines standards for integrating consumer devices and home area networks into the smart grid. Consequently, SEP 2.0 includes support for Wi-Fi (IEEE Std 802.11), Bluetooth (IEEE Std 802.15), Ethernet (IEEE Std 802.3), and broadband over power lines (IEEE Std 1901).

SEP 2.0 operates over both User Datagram Protocol (UDP) and TCP/IP, with support for both IPv4 and IPv6 (though it can also operate over a serial link using a hardware translator). Clientserver communications are conducted over HTTPS; payloads are encoded in XML. In addition to encryption, the SEP 2.0 specification addresses many of the authentication, authorization, and accounting requirements lacking in other protocols.

California investor-owned utilities adopted the Common Smart Inverter Profile (CSIP) as the profile specifying the use of IEEE Std 2030.5 for DER interconnection with the utility grid (Lai et al. 2017). CSIP-compliant equipment must have a device certificate installed that chains back to the root certificate authority. The SunSpec Alliance is responsible for the certification program for DER equipment, including the certificate authority, communications probing routine, and certifying test (SunSpec 2018). 
Each server maintains an access control list of clients that are authorized to communicate and access certain resources. If a device is on the list for a certain resource, it is authorized and has access to that resource; otherwise, it does not. Permissions might include the authority to read, write, and control. An aspect of access control is that different resource information might be presented based on the identity of the client making the request (i.e., role-based access control).

\subsubsection{Optional Communications Protocols}

During the development of the interoperability clause in IEEE Std 1547-2018, considerable discussion was given to communications protocols. Ultimately, the three listed were chosen; however, the standard allows DERs to use any communications protocol as long as the DER supports at least one of those specified. Informative Annex D in IEEE Std 1547-2018 notes IEC 61850 as being one such optional protocol, with DER-specific information models and guidance on DER functions specified in IEC 61850-7-420.

\subsection{Communications Architecture}

Experts suggest that direct control by utilities of many DER systems - even in the thousands - is not feasible (Cleveland and Lee 2013; IEC 2016). Instead, a hierarchical approach to utility communications and interaction with DERs is proposed. At the local level, DER systems manage their own generation (and storage) activities autonomously, based on local conditions, preestablished settings, and interconnection requirements. Subsequent levels might include facilities or customer energy management systems (EMS), retail energy providers or marketbased aggregators, area EPS operators (AEPSO) or distribution systems operators (DSO), and bulk power system (BPS) operations or energy markets. Although different scenarios and data requirements describe different hierarchical levels, it is valuable to consider this architecture to communications and interoperability. The scope of IEEE Std 1547-2018 describes the requirements and capabilities for the local DER communication interface only (see Figure 2).

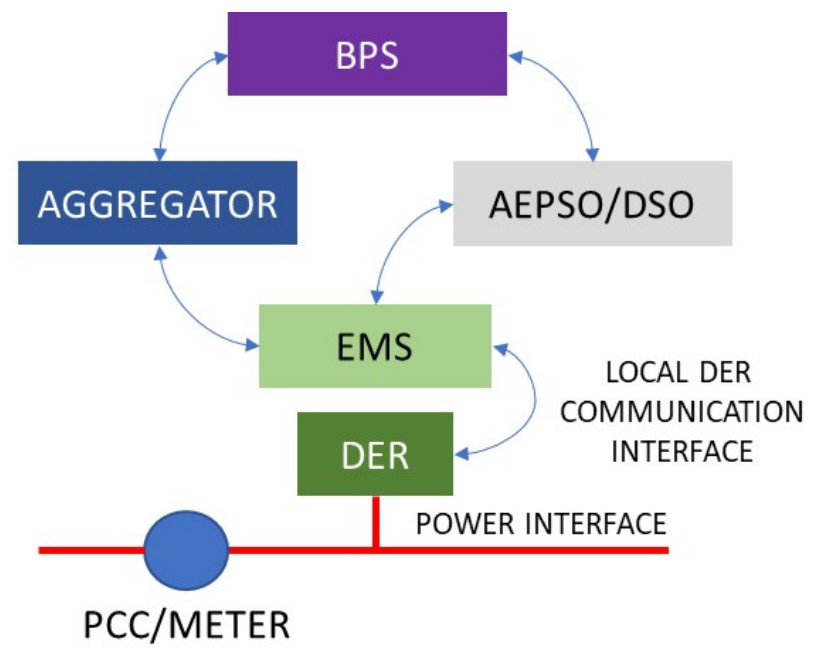

Figure 2. Hierarchical architecture for DER communications 


\section{Summary of Interoperability Requirements in IEEE Std 1547-2018}

IEEE Std 1547-2018 mandates interoperability capabilities; however, how they are implemented, recorded, and reported is determined by local jurisdictions and should be addressed in the relevant processes and procedures (such as interconnection agreements).

Two types of information are available at the communications interface: status and steady-state measurements.

\subsection{Information Exchange (Clause 10)}

Mandatory information elements capable of being exchanged at the communications interface fall into the following four categories:

- Nameplate information: This information describes the characteristics of the DERs when installed. Minimum parameters are specified and described in the standard. These include power and reactive power ratings, normal and abnormal performance categories, voltage ratings, and unit identification (model, serial number, software version). This information can be read. ${ }^{16}$

- Configuration information: Under mutual agreement between the DER operator and the area electric power system operator, each rating element in the "nameplate information" (see Table 28, IEEE Std 1547-2018) can be adjusted to an as-configured value. Note: Configuration settings are not intended for continuous, dynamic adjustment.

- Monitoring information: This information describes the present operating conditions of the DER. This information can be read.

- Management control information: This type of information allows for updates to the DER's functional and mode settings. This information can be read or written.

\subsection{Management Control Capability Requirements}

IEEE Std 1547-2018 requires DERs to have the capability to respond to external ${ }^{17}$ control using four functions: disable permit service, limit active power, change functional mode, and change parameters for control and protective functions.

Although these responsive capabilities are required, the decision regarding whether and how to use them is left to the area EPS operator.

\footnotetext{
${ }^{16}$ Read means capable of being displayed. Written means capable of being modified or adjusted. IEEE Std 1547.12020 uses the terms read-only and read-write.

17 The external input could come through a manual DER control panel or through the communications interface.
} 


\subsection{Security Considerations}

IEEE Std 1547-2018 recognizes the criticality of cybersecurity for DERs but defers to regulatory requirements and/or utility technical specifications or interconnection operating agreements and does not mandate specific cybersecurity requirements at the DER interface (IEEE 2018). ${ }^{18}$

With input from the AGIR, the area EPS operator should consider the degree of risk associated with various DER technologies and applications in determining cybersecurity requirements.

Informative Annex D of IEEE Std 1547-2018 (IEEE 2018) notes:

The security requirements associated with DER entail both local physical access and remote network access. The level of security required is proportional to the risk associated with a breach of the system and potential impact on other parts of the system.

The types of risks stakeholders should consider include the following:

- Physical and front-panel security:

- IEEE Std 1547-2018 does not prescribe specific requirements for securing the physical or digital communications interface. The following guidance is from the State of Minnesota (2020): “The DER operator should provide a reasonable level of security for the DER controls and devices from operation by intruders. The area EPS operator may specify additional physical security requirements in its interconnection agreement."

- Network security:

- The network security requirements for the DER operator are not specified by IEEE Std 1547-2918, but they are likely to be described in utility interconnection or operating agreements. These requirements and implementation details could differ among area EPS operators and are expected to evolve over time to maintain security in an environment of constantly changing threats (State of Minnesota 2020).

- Communications interface security:

O Though not directly specified in IEEE Std 1547-2018, when information is exchanged through the communications interface, consideration should be given to protect access to information by securing the interface. Specific requirementsincluding test and verification procedures, where practical - might be included in the utility interconnection or operating agreement (State of Minnesota 2020).

\footnotetext{
${ }^{18}$ On March 5, 2020, IEEE approved Project Authorization Request P1547.3 - Guide for Cybersecurity of Distributed Energy Resources Interconnected with Electric Power Systems to update IEEE Std 1547.3-2007.
} 


\subsection{Test and Certification}

Interoperability tests are specified in IEEE Std 1547.1 Clause 6 (IEEE 2020). Tests are conducted using the local DER communications interface, ${ }^{19}$ which is expected to be available at all times when the DER is operating (IEEE 2018). The interoperability requirements (e.g., information exchange) for each functional interconnection requirement the DER is capable of are tested using every communications protocol the DER supports, including testing adjustable settings. To streamline the testing process, the testing agency has the option to perform interoperability tests in parallel with the functional tests.

Overall, the formal testing requirements in IEEE Std 1547.1 verify that (1) the local DER communications interface can actually perform all read/write operations for the relevant nameplate, configuration, monitoring, and management functions of the DER; and (2) the DER responds appropriately within the communications time specified in the requirements.

Note that for the interoperability requirements specified in IEEE Std 1547, as tested using the procedures in IEEE Std 1547.1 and ultimately certified by a testing agency, the scope is limited to the DER. The upstream path from the DER to the entity communicating with the DER is out of scope of the standard itself (Figure 3). In practical terms, however, interoperability should be defined for the upstream path as well. Stakeholders in each local jurisdiction have the option to define that as they please. To date, California is the only U.S. state that has mandated a statewide approach to using the new DER interoperability capabilities - at least for inverter-based DERs. Specific guidance is given in the CSIP, which implements IEEE Std 2030.5 as the default communications protocol for DER interconnection. Certification to CSIP is provided by the SunSpec Alliance by a variety of testing agencies. ${ }^{20}$

${ }^{19}$ IEEE Std 1547-2018 defines the local DER communication interface as "A local interface capable of communicating to support the information exchange requirements specified in this standard for all applicable functions that are supported in the DER. The word interface is defined as an "electrical or logical connection from one entity to another that supports one or more energy or data flows implemented with one or more power or data links" (IEEE 2018).

${ }^{20}$ For more information, see https://sunspec.org/2030-5-csip/. 


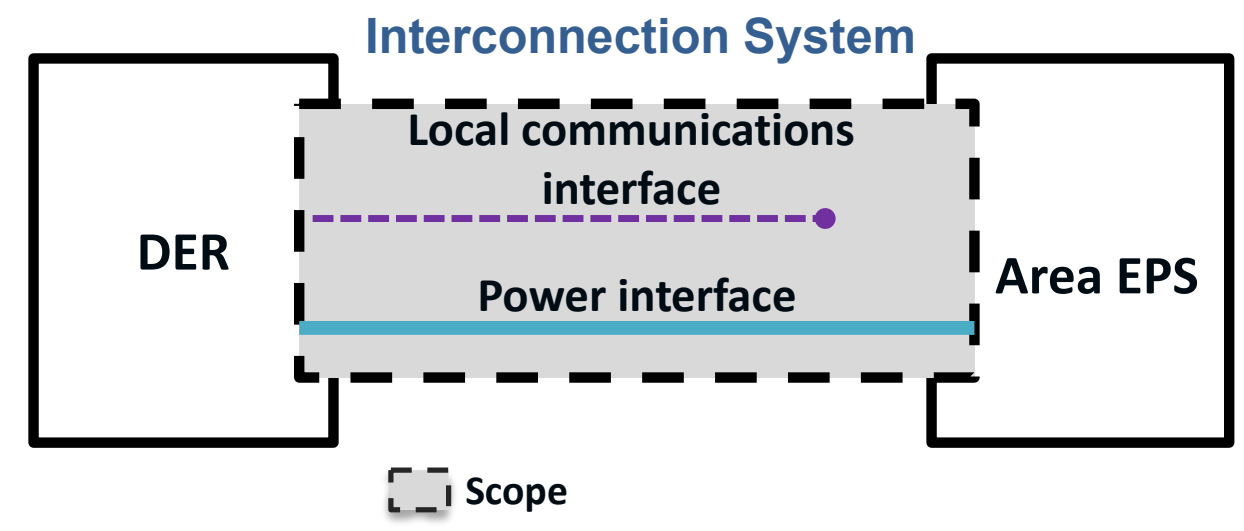

Image based on IEEE Std 1547-2018 (Fig. 1)

Figure 3. In-/out-of-scope 


\section{Considerations for the Utilization of Interoperability Capabilities}

IEEE Std 1547-2018 requires the capability to provide a communications interface, which is the basis for interoperability requirements. The communications interface can be used to exchange standardized information with the area EPS operator. The exchange of information allows the area EPS operator to perform monitoring and control functions necessary for the safe and reliable operation of the area EPS.

Per Section 10.1 of IEEE Std 1547-2018, the decision to use the communications interface or to deploy a communications network is determined by the area EPS operator. Given existing and future DER integration needs, as well as the differences among various area EPS operator's systems, no uniform set of standards is defined for requiring the use of the communications interface.

\subsection{Interoperability of Distributed Energy Resources and What?}

IEEE Std 1547 provides a standardization of the local DER communications interface and protocols, but it does not specifically address the external communications channel. These decisions are left for the AGIR, area EPS operator, and bulk power system stakeholders (i.e., regional reliability coordinator, balancing authority).

The North American Electric Reliability Corporation (NERC) recommends that these stakeholders will need to provide appropriate, coordinated guidance on policies, protocols, and mediums of communication (NERC 2020) and outlines the following topics for consideration:

- What are the use cases for external communications?

- What is the expected timeline for when a communications and control system might be needed?

- What performance level(s) might be required of the communications system to support specific applications?

- What are the parameters of the communications networks and architecture involved? For example, what standards will be applied? Who will own and operate each level of the communications system architecture?

- Which DERs (e.g., types, size) will be integrated into this standardized communications interface?

\subsection{Utility Requirements for Monitoring and Control}

When communications are required to the DER, the DER operator might be responsible for furnishing the communications channel from the area EPS operator's applicable system(s) to the DER and/or the meters. The forms of communication (cellular, radio, etc.) are determined by the area EPS operator. Additional details on communications requirements are typically specified in the area EPS operator's interconnection agreement or utility technical specifications or operating agreement. Additional details might include communications performance requirements, such as latency of exchanged information, periodicity, reliability of communications channels, and volumes of data. 


\subsection{Aggregators/Distributed Energy Resource Grid Services Providers}

Traditionally, utility-scale DER projects and those with special interconnection agreements (e.g., energy market participation) have been required to have external communications and remote controls to interface with the system operators. Unfortunately, these capabilities have not yet been standardized by the industry.

Because communications services will likely be provided by third-party aggregators that control numerous DERs, interconnection requirements or agreements that address the aggregator relationships should be considered by the AGIR. The recent Federal Energy Regulatory Commission Order 2222 (FERC 2020) cites the following comments from the New York Independent System Operator Indicated Transmission Owners, which might be informative:

“... any interconnection agreement for a distributed energy resource participating in an aggregation must demonstrate the ability of an individual distributed energy resource to (1) participate in an aggregation; (2) communicate essential information to the distribution system operator and RTO/ISO using RTO/ISO communication and operating protocols, as appropriate; and (3) meet RTO/ISO performance standards."

\subsection{Bulk Power System Data Needs}

Notably, in the voltage and frequency ride-through requirements, IEEE Std 1547-2018 recognizes and responds to the potential for DERs connected to the distribution system to affect planning and operations at the bulk power system level. A key implication of this understanding includes considerations for collecting and sharing DER data with entities responsible for modeling and planning the bulk power system.

NERC recommends that the regional entities-i.e., the Western Electricity Coordinating Council (WECC), Southeastern Electric Reliability Council, Midwest Reliability Organization, Northeast Power Coordinating Council, Reliability First, Texas Reliability Entity - consider and include DER data and information in their regional criterion for data requirements. NERC cites WECC's Steady State and Dynamic Data Requirements (WECC 2013) as an example of when these requirements should be included.

According to NERC (2017), examples of DER data categories that are important to support the adequate modeling and assessment of bulk power system reliability issues include the following:

- DER type (i.e., PV, wind)

- DER MVA rating

- Relevant energy production characteristics (i.e., active tracking, fixed tilt, energy storage characteristics)

- DER operating power factor and/or reactive and real power control functionality, PCC voltage

- DER location - behind the meter/in front of the meter

- Date the DER went into operation. 
In 2018, NERC updated their recommendations for DER data collection (NERC 2018). According to NERC, entities ${ }^{21}$ that might need to consider the effects of DERs include distribution providers, balancing authorities, transmission operators, transmission planners, and planning coordinators.

\subsubsection{Balancing Authorities}

Balancing authorities require knowledge of DER output and operating characteristics to adequately forecast load and operating and contingency reserve levels. NERC states that balancing authority operations can be enhanced with distribution (area EPS operators) load, net load, DER forecasts, as well as DER sensitivity to changing weather. Apart from this modelbased information, NERC recommends that balancing authorities obtain the following set of contextual DER data:

- DER aggregation on both a substation or wider area basis

- DER active power capability on both a substation and aggregated basis

- DER resource ramp rates in watts per minute (both ramp-down and ramp-up).

\subsubsection{Transmission Operators}

Because transmission system load is typically measured at the transmission-distribution transformer, distribution load might be offset by DER generation. Also, because transmission voltage and power flow, voltage control, and post-contingency conditions are all affected and vary by system loading, the transmission operator's real-time situational awareness of the transmission system and responses to contingencies could be negatively affected. Apart from certain model-based information (e.g. load, net load, and DER forecasts), NERC recommends that transmission operators obtain the following set of contextual and nameplate DER data:

- Aggregate nameplate capacity of DERs at each load bus

- Voltage and frequency ride-through capabilities of DER (abnormal performance categories assignment)

- Category III momentary cessation voltage threshold

- Voltage control capabilities

- Frequency control capabilities and settings

- Automatic restoration capability and settings.

\subsubsection{Transmission Planners and Planning Coordinators}

Transmission planners and planning coordinators use very detailed models of the bulk power system that include the aggregate load components of the distribution system. If DERs and distribution load are netted (i.e., load minus DER contribution), certain critical bulk power system impacts might be masked, including the response to off-nominal frequency and voltage, pre- and post-contingency response to disturbances, and transient response. Apart from certain model-based information (e.g., load, net load, and DER forecasts), NERC recommends that

\footnotetext{
${ }^{21}$ See NERC entities defined in the "Glossary of Terms," https://www.nerc.com/pa/Stand/Glossary\%20of\%20Terms/Glossary of Terms.pdf.
} 
transmission planners and planning coordinators obtain the same set of contextual and nameplate DER data listed in Section 3.4.2 for transmission operators.

\subsection{Screening Criteria for Monitoring and Control}

When monitoring and/or control might be required, industry has proposed a list of screening criteria (Joint Utilities of New York 2017).

- Control might be needed to increase PV hosting capacity on certain feeders.

- Recloser (control) at the PCC might be required to satisfy anti-islanding requirements.

- Monitoring will be required for PV systems less than $50 \mathrm{~kW}$ that only marginally pass or fail a given standardized interconnection requirements screen; similarly, "borderline" systems rated from $50 \mathrm{~kW}-300 \mathrm{~kW}$ might require control.

- Monitoring will be required if the PV system is subject to independent system operator requirements, and control might also be required.

- Control might be required for proposed PV systems that exceed a certain capacity limit (to be determined) of the minimum daytime load to address concerns related to voltage, thermal limits, and power system protection.

- Monitoring and control might be required if the proposed PV system exceeds a certain (to be determined) capacity of the minimum daytime load to address concerns related to voltage, thermal, and power system protection.

- Monitoring and control might be required if the proposed PV system is on a single-phase line section and creates a greater than (to be determined) imbalance a the next upstream three-phase location.

When information exchange through the communications interface is required by the area EPS operator, the IEEE Std 1547-2018 interoperability parameters are expected to be available for use. Specifically, these parameters are detailed in the subclauses associated with IEEE Std 1547-2018, Section 4.6: Control capability requirements, including the capability to disable permit to service, the capability to limit active power, and the execution of mode and parameter changes.

\subsection{Summary of Considerations}

Interoperability, communications, and information protocols were not addressed in the prior (2003) version of IEEE Std 1547 and were left for utilities and DER developers to negotiate based on system needs. This created challenges for the equipment suppliers as well as for utilities. IEEE Std 1547-2018 brings new requirements for communications, specifying protocols that must be available, and what information can be read and written — at the local

communications interface.

If interoperability capabilities are enabled, interconnection rules will need to specify which DERs will be required to integrate with communications systems and which communications protocol the area EPS operator will use at the DER communications interface.

IEEE Std 1547-2018 provides a standardization of the local DER communications interface and protocols, but it does not specifically address the external communications channel. These decisions are left for the AGIR, the area EPS operator, and the bulk power system stakeholders 
(i.e., regional reliability coordinator, balancing authority). Based on lessons learned from the implementation of interoperability requirements in other jurisdictions, an implementation profile such as the CSIP could provide beneficial guidance for both utility and DER operators. 


\section{References}

Adams, Heather. 2017. "Joint Utilities of New York: Monitoring and Control Discussion." Presented at the Interconnection Technical Working Group, January 18, 2017.

Cleveland, Frances, and Annabelle Lee. 2013. Cyber Security for DER Systems. Palo Alto, CA: Electric Power Research Institute.

Federal Energy Regulatory Commission (FERC). 2020. Participation of Distributed Energy Resource Aggregations in Markets Operated by Regional Transmission Organizations and Independent System Operators, Order 2222 FERC $\uparrow$ 61,247.

Institute of Electrical and Electronics Engineers (IEEE). 2018. IEEE Std 1547-2018 - IEEE Standard Conformance Test Procedures for Equipment Interconnecting Distributed Energy Resources with Electric Power Systems and Associated Interfaces. Piscataway, NY.

- 2020. IEEE Std 1547.1-2020 - IEEE Standard for Interconnection and Interoperability of Distributed Energy Resources with Associated Electric Power Systems Interfaces. New York, NY.

International Electrotechnical Commission (IEC). 2012. Distributed Energy Management (DER): Advanced Power System Management Functions and Information Exchanges for Inverter-Based DER Devices, Modelled in IEC 61850-90-7. Technical Committee 57 Working Group 17.

- 2016. IEC TR 62351-12 - Technical Report: Power systems management and associated information exchange - Data and communications security - Part 12: Resilience and security recommendations for power systems with distributed energy resources (DER) cyber-physical systems. Geneva, Switzerland.

Joint Utilities of New York - Interconnection Technical Working Group. 2017.

“03/29/17 ITWG Meeting Follow-Ups - Monitoring and Control Screens." April 28, 2017. http://documents.dps.ny.gov/public/Common/ViewDoc.aspx?DocRefId=\%7B472888AD-7B8F4938-9FE3-9D3F6D38FCAA\%7D.

Lai, Christine, Nicholas Jacobs, Shamina Hossain-McKenzie, Cedric Carter, Patricia Cordeiro, Ifeoma Onunkwo, and Jay Johnson. 2017. Cyber Security Primer for DER Vendors, Aggregators, and Grid Operators. Albuquerque, NM: Sandia National Laboratories, December 2017. http://sunspec.org/wp-content/uploads/2017/08/DERCyberPrimer-DraftforReview.pdf.

Modbus. 2018. MODBUS/TCP Security: Protocol Security. https://modbus.org/docs/MB-TCPSecurity-v21_2018-07-24.pdf.

North American Electric Reliability Corporation (NERC). 2017. Distributed Energy Resources: Connection Modeling and Reliability Considerations. Washington. D.C. 
- 2018. "Technical Brief on Data Collection Recommendations for Distributed Energy Resources." March 29, 2018.

https://www.nerc.com/comm/Other/essntlrlbltysrvcstskfrcDL/DER_Data_Collection_Tech_Brief 03292018_Final.pdf

2020. Reliability Guideline: Bulk Power System Reliability Perspectives on the Adoption of IEEE 1547-2018. Washington, D.C.

State of Minnesota. 2020. "State of Minnesota Technical Interconnection and Interoperability Requirements (TIIR).” January 22, 2020.

SunSpec Alliance. 2018. Common Smart Inverter Profile: IEEE 2030.5 Implementation Guide for Smart Inverters. San Jose, CA: March 2018.

U.S. Congress. 2005. “Energy Policy Act of 2005.” Sec. 1234. Washington, D.C.

U.S. Department of Energy (DOE). 2015. United States Electricity Industry Primer. Washington, D.C.: July 2015.

Western Electricity Coordinating Council (WECC). 2013. Steady State and Dynamic Data Requirements MOD-(11 and 13)-WECC-CRT-1 Regional Criterion. Salt Lake City, UT: June 26, 2013. 